\title{
TÉCNICAS CONSTRUCTIVAS EN LAS TERMAS ROMANAS DE CAMPO VALDÉS (GIJÓN): EL MATERIAL LATERICIO ${ }^{1}$
}

\author{
POR \\ CARMEN FERNÁNDEZ OCHOA y MAR ZARZALEJOS PRIETO \\ Universidad Autónoma de Madrid
}

\section{RESUMEN}

Se aborda en este trabajo el análisis del empleo de lateres coctiles en la construcción de las termas romanas de Campo Valdés (Gijón). Este estudio es una contribución al conocimiento de la edilicia hispanorromana en general y, de manera particular, al de la técnica constructiva en los complejos termales.

\section{SUMMARY}

In this paper we analyze the use of lateres coctiles in the building of the Roman baths at Campo Valdés in Gijón (Spain). This study is meant as contribution to the knowledge of Roman building techniques in general and, as a print of detail, to the knowledge of bath building techniques and materials.

\section{INTRODUCCIÓN}

La ciudad de Gijón contó en época romana con un edificio termal de carácter público, cuyos vestigios se conocen parcialmente desde principios de siglo (Somoza, 1908; Alvargonzález, 1965). En 1990, dentro del Proyecto Gijón de excavaciones arqueológicas, se procedió a la reexcavación del monumento con el fin de realizar una revisión sistemática de los restos conservados y recuperarlos para su exhibición pública. Recientemente en el marco de la exposición Astures se inauguraron las instalaciones museísticas que albergan las ruinas del complejo termal (Fernández Ochoa, dir., 1995).

Las termas romanas de Gijón están ubicadas en la plaza de Campo Valdés, en la falda suroriental de la península de Sta. Catalina, que actualmente ocupa el popular barrio de Cimadevilla. Este monumento constituye, junto con la muralla tardorromana y la factoría de salazones de la Plaza del Marqués, la evidencia arqueológica más relevante

\footnotetext{
1 Este trabajo se enmarca dentro de nuestro proyecto «Termas públicas y baños privados en la Hispania romana», subvencionado por la DGYCIT (PS92-0025) y actualmente en curso.
}

de la romanidad de Gijón (Fernández Ochoa, 1992; 1993; 1994).

La aportación que vamos a realizar en este estudio se refiere a la utilización de uno de los elementos más característicos de las termas romanas, el material latericio, cuyo uso en los sistemas de calentamiento y en los paramentos de las habitaciones calefactadas representa un rasgo específico de estas instalaciones. A través de este análisis pretendemos cubrir un aspecto a menudo olvidado en los estudios sobre las termas romanas hispanas, cuya deficiencia ya hemos manifestado en otro lugar (Fernández Ochoa, Morillo, Zarzalejos, 1995, e.p.).

El análisis e interpretación del uso de los lateres coctiles en las diversas estancias exhumadas en las termas de Gijón será abordado teniendo en cuenta la interpretación funcional del edificio según se ha dado ya a conocer a la comunidad científica (Fernández Ochoa (Dir.), 1995) (fig. 1).

\section{EL USO DE MATERIAL LATERICIO EN LAS TERMAS DE GIJÓN}

Como es bien sabido, las estructuras calefactadas son las que proporcionan mayor información respecto al uso del material latericio en los edificios termales. El empleo de este tipo de material se constata asimismo en otras partes de las termas, como paramentos e infraestructuras de alcantarillado, conducción y desagüe. El orden de la exposición de datos que sigue considera en primer lugar las habitaciones calefactadas, de acuerdo con los siguientes elementos constitutivos:

- Praefurnia

- Pasos de calor

- Area

- Elementos de sustentación: pilae y arquillos

- Revestimientos refractarios parietales

- Suspensurae

- Concamerationes 


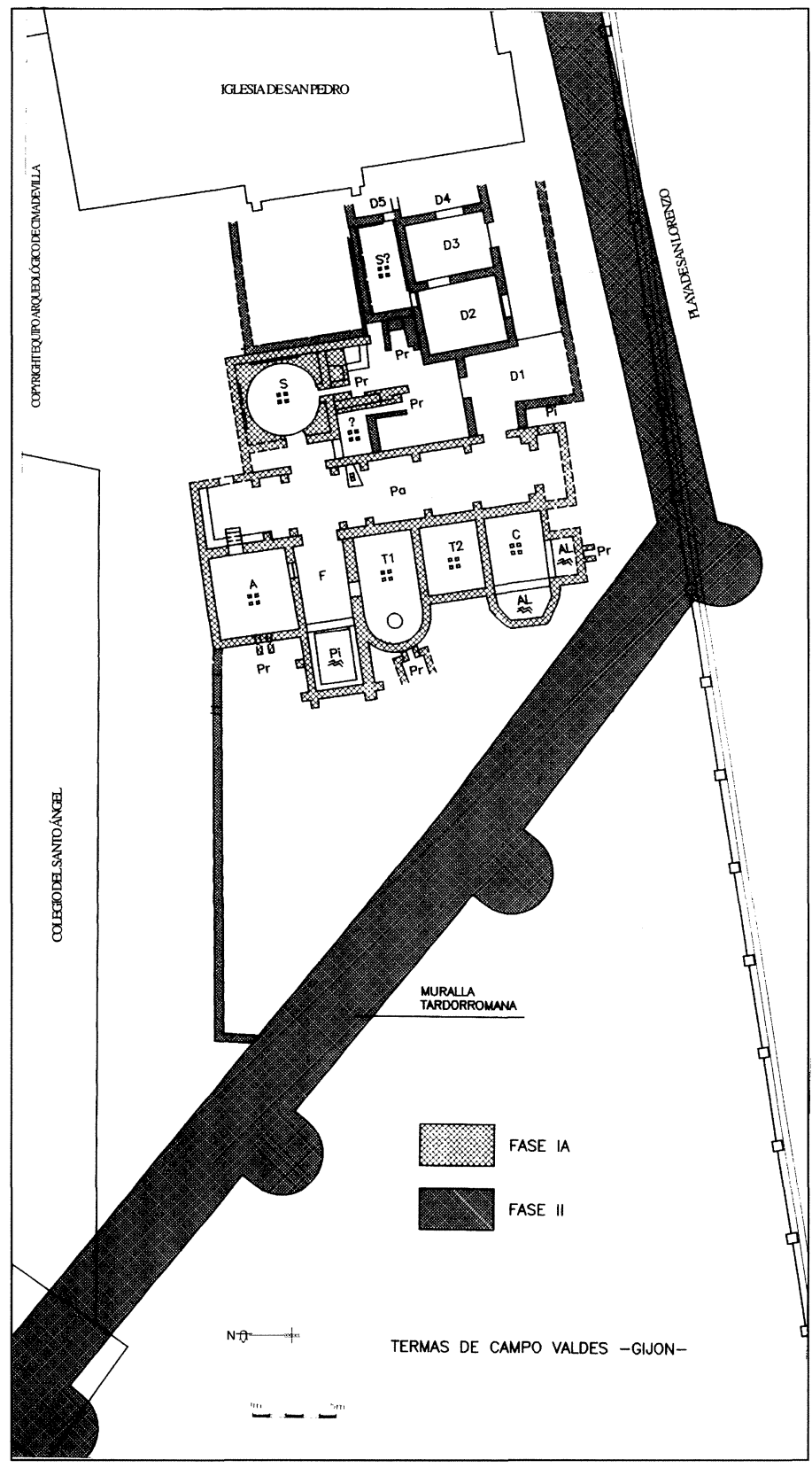

Figura 1.-Planta de las termas con indicación de sus fases constructivas.

\section{a) Hipocaustos}

\section{El primer proyecto}

El apodyterium (A) no conserva vestigios de praefurnium, si bien se observaron durante el proceso de excavación restos de tierra quemada en la pared oeste, que, junto a la interrupción constatada en dicho muro y la inclinación de la estancia hacia el oeste (entre 4 y $7 \mathrm{~cm}$ sobre la cota media), inducirían a pensar que efectivamente existió un hogar en este punto. La construcción del area se realizó en opus signinum de buena calidad, de superficie homogénea con alta composición en latericio pulverizado.

La suspensura reposó sobre un entramado de arquillos, compuesto por dieciséis filas de seis arcos en sentido E-W. Se conservan restos de veintiséis arranques además de las huellas de otros hasta alcanzar un total de sesenta. Se trata de arcos de medio punto, uno de los cuales se ha conservado completo en la esquina NW (fig. 2). Los ladrillos empleados en su fábrica son de tipo cuneati, con medidas medias de $19 \times 12 \times 3,5 / 4,8$ $\mathrm{cm}$, salvo en la intersección de dos arcos, donde se ha localizado un bessalis de $21 \times 20 \times 4 \mathrm{~cm}$.

La pared este conserva un revestimiento refractario integrado por 18 ladrillos bessales $(20 \times 19 \times 7 \mathrm{~cm}) \mathrm{en}$ la base, dispuestos en vertical. Del alzado subsisten siete hiladas de ladrillos rectangulares indeterminados $(18,5 / 19 \times 12 / 13 \times 4 / 5 \mathrm{~cm})$. En la pared sur, bajo el acceso al frigidario, se documenta un refuerzo refractario constituido por tres series verticales de cinco hiladas de ladrillos de tipo lydion $(28 / 29,5 \times 40 / 44 \times 5,5 \mathrm{~cm})$.

La suspensura está compuesta por grandes imbrices $(50 \times 10 \times 26 \mathrm{~cm})$ cubiertas por dos capas de opus signinum. Se conserva in situ uno de ellos en la pared este sobre el muro refractario.

El primer tepidarium (T1) presenta restos de la cimentación de una pequeña cámara cuadrangular (propnigeum) que precede al praefurnium; éste se configura como un canal exterior relacionable con 


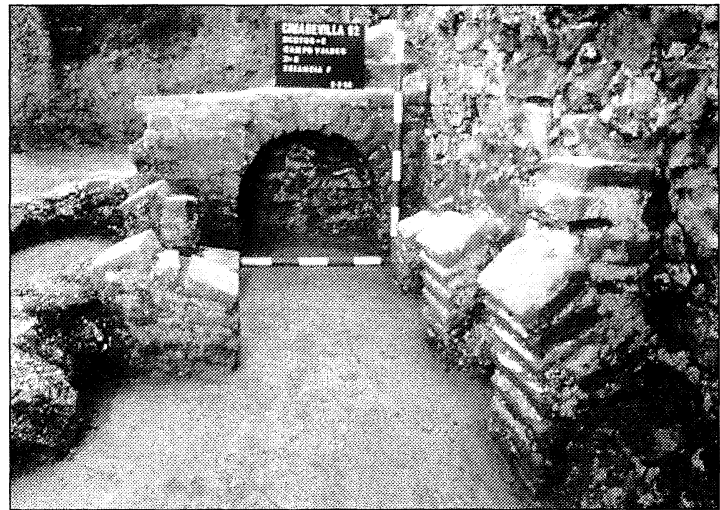

Figura 2.-Detalle de los arquillos conservados in situ en el apodyterium (A).

el tipo III de Degbomont (Degbomont, 1984, 62). El latericio aplicado a la realización de los muretes del praefurnio es mixto: lydiones (29/30 x 44/45 x 5/6 $\mathrm{cm})$ y pedales $(31 \times 30 \times 5,5 \mathrm{~cm})$ (fig. 3$)$.

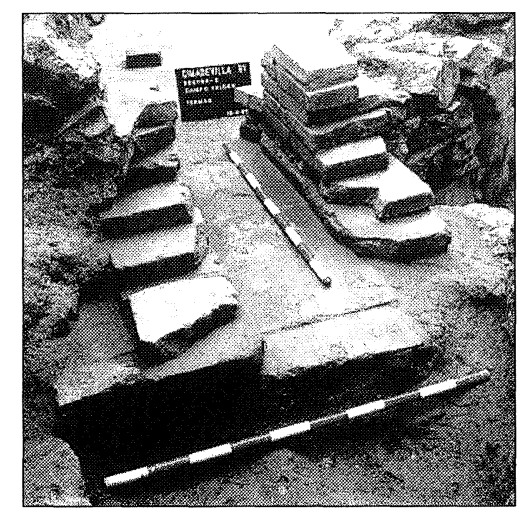

Figura 3.-Detalle del praefurnium del primer tepidarium (T1).

Pueden reconocerse dos pasos de calor hacia la habitación contigua por el sur. El mejor conservado presenta piezas de tipo lydion $(44 \times 30 \times 5,5 / 6 \mathrm{~cm})$, a las que se adicionan fragmentos de ladrillo de unos $20 \mathrm{~cm}$ hasta completar la anchura del muro (fig. 4).

El area está realizada con opus signinum de buena calidad y ofrece una inclinación hacia el suroeste que se aproxima a los $10 \mathrm{~cm}$ sobre la cota media.

Los elementos de sustentación son pilae, distribuidas en diez filas en sentido E-W de seis hileras cada una. No se conserva ninguna, pero sí su impronta marcada sobre el pavimento, hecho que permite cifrar las dimensiones del latericio empleado

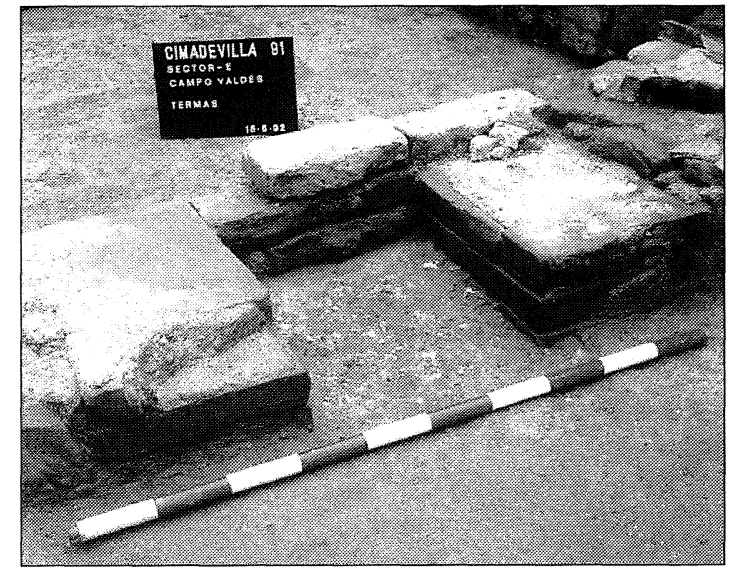

Figura 4.-Detalle de uno de los pasos de calor entre los dos tepidaria (T1 y $\mathrm{T} 2)$.

en torno al módulo bessalis $(18,5 / 19 \times 6,5 / 7,5 \mathrm{~cm})$. La distancia entre las pilas es de $48 \mathrm{~cm}$ aproximadamente. A juzgar por el tamaño de las improntas, puede asegurarse que carecieron de base por lo que indistintamente podrían corresponder a los tipos A, H o J (Degbomont, 1984, 100).

La zona absidiada de esta estancia conserva restos de un revestimiento refractario, constituido por ladrillos bessales $(18 \times 19 \times 6,5 / 7 \mathrm{~cm})$.

No se documentan vestigios de la suspensura, pero es posible su reconstrucción con piezas bipedales teniendo en cuenta los módulos de distancia entre las pilae ${ }^{2}$.

No ha proporcionado este ambiente indicio alguno que permita identificar el empleo de concamerationes.

La siguiente sala, considerada por nosotros como un segundo tepidario (T2), apenas conserva restos de su sistema de calefacción. En todo caso, se trata de una estancia calentada indirectamente desde los ambientes contiguos (T1 y C), cuyos pasos de calor se describen en cada una de las estancias correspondientes. El area se pavimentó con un opus signinum del que restan únicamente las capas inferiores de este preparado hidráulico.

Como elementos sustentantes del suelo de la habitación, sólo es posible apuntar la existencia de un ladrillo bessalis $(18 \times 18 \times 7 \mathrm{~cm})$ sobre el área en el ángulo NE, que correspondería a un soporte de la modalidad de pilae. El deterioro que presenta la

${ }^{2}$ De hecho, en la reconstrucción con fines didácticos realizada en esta estancia dentro del actual Museo-yacimiento de las Termas de Campo Valdés, se ha adoptado este patrón latericio, dado que la distancia entre el punto central de cada pila y su consecutiva se aproxima a los sesenta centímetros. 
pavimentación del area impide reconocer las improntas de la sucesión de pilas y, en consecuencia, establecer un cálculo aproximado de sus distancias y su disposición espacial en este ambiente.

$\mathrm{El}$ caldarium (C) no ha proporcionado restos identificables de un praefurnium, debido posiblemente a las alteraciones sufridas por el extremo sur del complejo termal en época moderna ${ }^{3}$. En la reconstrucción hipotética de esta sala, hemos considerado la posible existencia de un praefurnium en el extremo sur de la habitación, quizá asociado a un segundo alveus, siguiendo un esquema frecuentemente repetido en los complejos termales de Hispania y otras zonas del Imperio (Nielsen, 1990, passim).

Los pasos de calor radicados entre T2 y C están realizados con ladrillos de tipo lydion (43/44 x 27/30 x $6 \mathrm{~cm}$ ) y se recurrió al mismo sistema de adecuación con el grosor del muro ya comentado en la descripción de los pasos de calor existentes entre T2 y T1.

El area del caldarium presenta una pavimentación de opus signinum que ha perdido en parte su acabado superficial.

La suspensura de esta estancia está apoyada sobre pilae de ladrillos bessales $(18 \times 18 \mathrm{~cm})$, cuya impronta permite identificar su distribución en ocho hileras (este-oeste) de cinco pilas (norte-sur) (fig. 5). En el espacio absidiado en el que se ubicó el

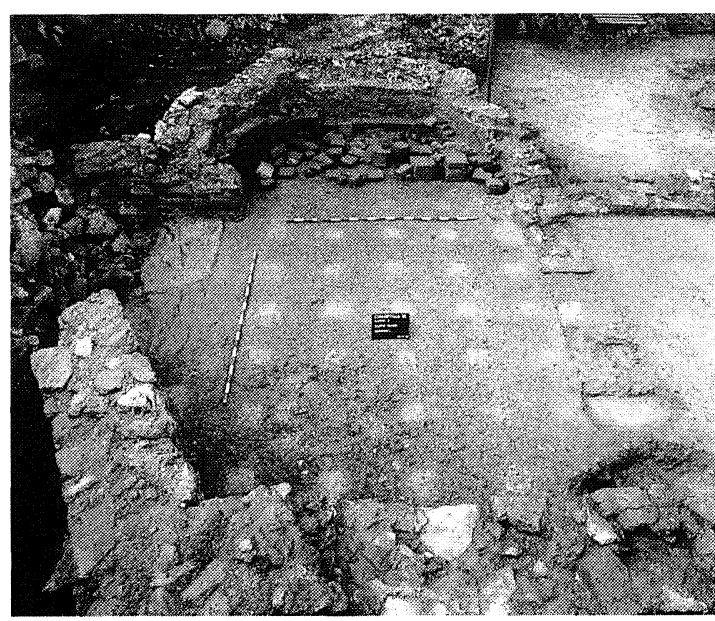

Figura 5.-Improntas de las pilae y pasos de calor del caldarium $(\mathrm{C})$.

\footnotetext{
${ }^{3}$ La excavación arqueológica puso de manifiesto la existencia de graves deterioros producidos por la erección de estructuras murarias de protección ante las acometidas del mar. En el transcurso de nuestros trabajos en este sector se han recuperado materiales cerámicos y numismáticos de cronología moderna (siglos XVIII-XIX), que corroboran las noticias acerca de la financiación de la obra por parte de la familia Jovellanos (Bonet, 1970).
}

alveus, se conservan in situ tres hileras (este-oeste), dos de ellas de cuatro pilas y una tercera de cinco (norte-sur). La distancia media entre los elementos de suspensión es de unos $48 \mathrm{~cm}$.

El caldario ha proporcionado vestigios de un muro refractario en cuya fábrica se advierte el empleo de ladrillos bessales $(17,5 / 18,5 \times 17,5 / 18,5 \times$ $6,5 / 7 \mathrm{~cm})$ en la mitad sur y cuneati $(21 / 22 \times 15 / 15,5$ $\mathrm{x} 4,5 / 5 \mathrm{~cm}$ ) en la porción norte.

La habitación no conserva restos de la suspensura. Una vez más es posible deducir, por el canon de distancia entre pilae, que ésta se configuró mediante grandes piezas bipedales.

Dentro del primer proyecto termal que venimos analizando, se produce una reforma que supuso el añadido de una nueva estancia al noreste del pasillo (Pa) y la modificación de la circulación inicial de estos espacios. El nuevo ambiente es una habitación circular, inscrita en un cuadrado que se puede interpretar como una sudatio (S). El hipocausto, cuyo buen estado de conservación permitió su exhibición pública a lo largo de los años, ha sido objeto de algún retoque desde su descubrimiento. Su praefurnium, clasificable dentro del tipo III de Degbomont, presenta lateres de distinto tamaño. Los tres reconocibles en la zona superior de los muretes corresponden al tipo lydion (41/46 x 22,5/28,5 x 4/5 cm), módulo con el que se relacionan también los de la base. Este hogar tiene acceso desde un patio de servicio, que será común a otros praefurnia asociados a estancias pertenecientes a la fase de ampliación que experimenta el complejo en el siglo II d.C. (fig. 1). Los restos conservados no posibilitan aventurar si en la fase constructiva inicial el praefurnium de la sudatio circular contó con prognigeum.

El area está pavimentada con opus signinum, sobre el que se levantan las pilae. Los apoyos suman un total de 36 ejemplares, en su mayor parte circulares, con basa y capitel de uno o dos pedales $(19,5 \times 19,5 \times 4 \mathrm{~cm})$ o con pedalis y bessalis en el caso de algunos capiteles, siguiendo los modelos B y $G$ de Degbomont. En el extremo occidental, en correspondencia con el acceso a este ambiente, se dispusieron dos arquillos realizados con cuneati. La clave de uno de ellos reposa sobre una pila de ladrillos circulares del tipo B de Degbomont. La hilera de pilae inmediatamente situada hacia el este combina el modelo circular con dos cuadradas en ambos extremos.

La suspensura se ha realizado con bipedales $(55 \times 55 \times 8 \mathrm{~cm})$, sobre los que se asentó el pavimento de opus signinum, conservado sólo en parte.

La estancia ha proporcionado vestigios de concamerationes, consistentes en tubuli latericii $(25 \mathrm{x}$ 
$42 \times 10 \mathrm{~cm}$ ) que resultan visibles en los lados norte $\mathrm{y}$ este.

\section{Ampliaciones y reformas}

A partir del primer tercio del siglo II d. C., el conjunto experimenta una ampliación hacia el este. Entre las nuevas estancias que se incorporan en el complejo balneario debemos mencionar dos en particular, provistas de hipocausto (S ? y $\mathrm{H}$ ?), dado que los ambientes restantes (D1, D2, D3 y D4) no cuentan con sistema de calefacción.

Atribuimos a la primera dependencia citada una función de sudatio. Esta interpretación se basa en las dimensiones reducidas de la habitación y el enorme potencial calorífico que las estructuras conservadas permiten reconstruir. Se trata de una estancia de forma rectangular, que cuenta con el hipocausto mejor conservado del conjunto gijonés (fig. 6).

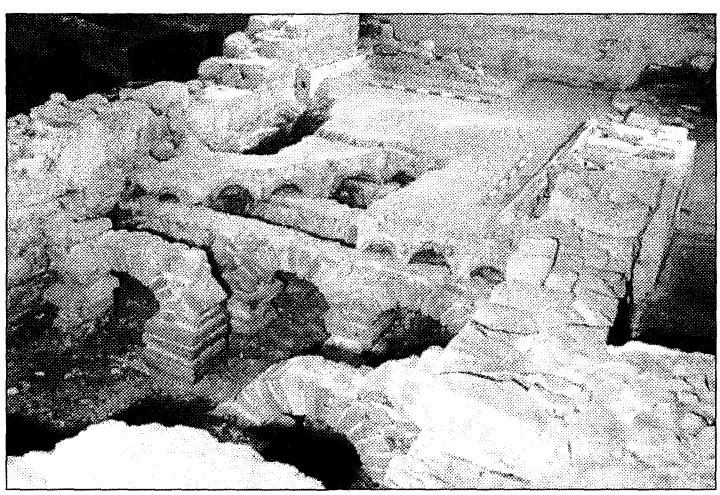

Figura 6.-Hipocausto de la posible sudatio rectangular.

E praefurnium está constituido por ladrillos irregulares y tegulae. Podría clasificarse dentro del tipo III de Degbomont y dispone de un propnigeum a la manera de una pequeña estancia delimitada por sendos muretes de mampostería de distinto grosor. El más grueso - $60 \mathrm{~cm}$ - se adosa a la pared de la estancia D2, probablemente para aislar las pinturas murales de la citada habitación del calor desprendido por el praefurnium. El arco del praefurnio está compuesto por pilas de cinco hiladas de ladrillos de diferentes tipos colocados horizontalmente (pedales $27 / 29,5 \times 4 / 5 \mathrm{~cm}$; lydion $26 \times 42 \times 5,5 \mathrm{~cm}$ y lydion dovelado $27,5 \times 44$ × 4/5 o 27,5 x 38 × 5,5 cm); por encima de ellos se coloca una tegula a modo de cuña cuya pestaña sirve de arranque al arco. Este último se formó con ladrillos pedales, pedales do- velados y cuneati. En la entrada del horno se documentó un lydion $(49 \times 32,5 \times 5 \mathrm{~cm})$, mientras que el conducto interno se compone de nueve ladrillos pedales $(27 / 31 \times 5 / 6 \mathrm{~cm})$ y un lydion $(27 \times 42 \times 5,5$ $\mathrm{cm})$. La bóveda está realizada con cuneati indeterminados de $19 \times 4 \mathrm{~cm}$ aproximadamente.

El area está pavimentada con opus signinum de acabado grueso y no es posible calibrar su inclinación respecto al praefurnium, dado que no se ha podido vaciar el extremo opuesto.

El sistema de suspensión elegido en este ambiente son los arquillos. Se distribuyen en diez hileras de tres arcos con una luz de 50 o $60 \mathrm{~cm}$; en este conjunto el rasgo más sobresaliente es el de la diversidad. En efecto, hemos identificado hasta tres procedimientos de construcción de las pilas de los arcos:

- pila de cuneati: se utilizan ocho ladrillos $\mathrm{cu}$ neati de $18 / 20 \times 14 \times 3 / 4,5 \mathrm{~cm}$.

- pila de ladrillos cuadrados: se emplea el módulo pedalis de $27 / 29 \times 5 / 6 \mathrm{~cm}$.

- pila de ladrillos troncopiramidales: los arquillos apoyan en plintos cerámicos en forma de ábaco. En su mayor parte presentan unas dimensiones próximas al módulo pedalis, si bien hay algunos ejemplares rectangulares.

Para la realización de las dovelas se aplicaron entre doce y quince ladrillos cuneati de dimensiones diversas $(18,5 / 19,5 \times 13,5 / 14 \times 4,5 / 5,5 \mathrm{~cm})$.

La suspensura está construida mediante grandes imbrices $(48 \times 21 \times 10 \mathrm{~cm})$ que apoyan sobre una lechada de argamasa mezclada con fragmentos de ladrillo, teja y piedra que rellena el tímpano. Sobre las imbrices se dispuso una primera pavimentación en opus signinum de tonalidad blanquecina, rico en cal (terrazo-signinum), con una fina moldura perimetral. En un segundo momento, se procede a una repavimentación realizada, esta vez, con un signinum más denso y rojizo a causa de la mayor proporción de latericio triturado. Las esquinas de la habitación se refuerzan con la clásica moldura en cuarto de bocel.

La habitación conserva restos de concamerationes, constituidas por tubuli latericii $(25,5 / 26 \times 10,5 /$ $12 \mathrm{~cm}$ ).

En cuanto a la segunda estancia calefactada, situada al sur de la sudatio circular (S), se trata de un espacio irregular formado por un pequeño ambiente cuadrado al que se adosa un apéndice piriforme por el oeste. El ambiente cuadrangular conserva restos de un hipocausto realizado con materiales latericios amortizados de las etapas anteriores. El praefurnium se localiza en el extremo suroriental de la estructura y está formado por dos muretes; el que está situado 
hacia el oeste presenta ladrillos rectangulares muy afectados por el calor y cuya medición exacta resulta difícil (10/12 x 18/22 x $5 \mathrm{~cm}$ aprox.). El pésimo estado de conservación del muro oriental impide obtener medidas precisas de los lateres que lo revisten. En el suelo de la estructura, entre ambos muretes, se hallaron dos lydiones $(43 \times 30 \times 5 \mathrm{~cm})$.

El area está realizada con opus signinum de mala calidad. Los elementos de sustentación son pilae constituidas por un sistema mixto resultante del empleo de materiales latericios reutilizados. Así, se combinan pilae con ladrillos cuadrados de tipo bessalis $(20 \times 21 \times 8 \mathrm{~cm})$ con un pedalis $(28 \times 28 \times 6$ $\mathrm{cm})$ a modo de basa; está documentada también la modalidad de columnilla de ladrillos circulares (18 $\mathrm{cm}$ de diám. x $6,5 \mathrm{~cm})$ con basa de pedalis $(28 \times 28$ x $5 \mathrm{~cm}$ ). Una última variedad está representada por pilas con una base pedalis $(28 \times 28 \times 5,5 \mathrm{~cm})$, sobre la que se conservan dos ladrillos redondos $(18 \mathrm{~cm}$ de diám. x 7/8 cm) y dos bessales $(20 \times 20 \times 6 \mathrm{~cm})$.

Se ha constatado la realización de un revestimiento refractario formado por cuneati $(18 \times 14 \times 5 /$ $6 \mathrm{~cm})$, que se halla adosado al muro occidental de esta pequeña estancia.

El apéndice piriforme conserva asimismo restos de hypocausis, formada por tres pilas centrales yuxtapuestas con ladrillos cuadrados de dimensiones comprendidas entre el módulo bessalis y el pedalis (26 $7 \mathrm{~cm}$ y $23 / 24 \times 7 \mathrm{~cm})$. Para la construcción del muro de la estancia se emplearon, además de la piedra caliza, fragmentos de tegulae, imbrices y ladrillos. Siguiendo un eje norte-sur se dispusieron dos chimeneas simétricas construidas con tegulae.

\section{b) Red DE ALCANTARILLADO Y ABASTECIMIENTO DE AGUA}

La red de conducciones detectada en la reexcavación de las termas de Campo Valdés corresponde en su mayor parte al sistema de desagüe de las estructuras exhumadas en la zona central del complejo, así como también de las edificaciones localizadas en el noreste, junto a la iglesia de San Pedro, que posiblemente formaron parte de las Termas ${ }^{4}$.

Las características constructivas de estos desagües son bastante uniformes: la base del canal está

${ }^{4}$ En 1994 se han practicado excavaciones de urgencia en la Plaza del Arcipreste Piquero y Avda. de la Salle que sacaron a la luz tres habitaciones con estructuras murarias y decoración parietal muy semejantes a las de las termas. Es probable que el edificio, en su ampliación, alcanzase estos espacios dada la certeza de que el complejo se prolongaba bajo la iglesia de San Pedro. realizada con una sucesión de tegulae. Se ha documentado el empleo de tejas de distintas dimensiones. Buena parte de las conducciones conservadas in situ presentan un módulo de $46 / 49$ x $37 \mathrm{~cm}$. Otras piezas más pequeñas $(46 \times 18 \mathrm{~cm})$ se han hallado fuera de contexto, salvo dos ejemplares de este mismo tamaño ubicadas en una conducción. Cualquiera que sea el módulo empleado, todas ellas se encastraron en la arcilla, delimitadas por una hilera de piedras trabadas con argamasa. La cubierta se compone de grandes lajas de caliza y en un solo caso - la conducción de traída de agua al frigidarioestá realizada con tegulae.

Escapan a esta tónica general tres canalizaciones. La primera de ellas funciona como desagüe de la piscina del frigidarium y en su base se emplearon ladrillos de tipo lydion $(38 \times 30 \times 5 \mathrm{~cm})$, material que posiblemente pudo servir para su cubrición, según se observa en el perfil este del frigidario (F). La segunda se encuentra localizada sobre la fábrica muraria del hipocausto de la sudatio circular. En la base se aplicaron ladrillos rectangulares a los que únicamente ha sido posible medir la anchura (33 $\mathrm{cm})$, mientras que los muretes laterales fueron construidos con ladrillos cuneati $(18,5 / 19 \times 14 \times 4 / 5,5$ $\mathrm{cm})$. Por último, habría que aludir a un pequeño tramo de conducción situado bajo el ambulacrum $(\mathrm{Pa})$, en dirección hacia el tepidarium (T1). La base está compuesta por tres imbrices de sección apuntada y una tegula en la zona cercana a la cara oeste del muro del citado tepidario. La cubrición se realizó con dos piezas rectangulares $(48 \times 24 \mathrm{~cm})$, con la cara vista plana y la interior cóncava, provistas de entalles para asegurar el encaje entre sí. Estas piezas no son frecuentes entre las conducciones conocidas en las termas hispanas.

Todas ellas confluyen hacia la zona de servicio situada entre la sudatio circular (S), el ambulacrum (Pa), la sudatio rectangular (S ?) y las dependencias D1 y D2. Desde este punto se encaminan hacia el mar orientando su salida bajo la dependencia D1.

Sólo ha sido posible identificar una conducción de abastecimiento. Arranca de un aljibe parcialmente conservado y que se encuentra adosado al muro norte del apodyterium (A). La base y la cubierta de la canalización se realizaron con tegulae de gran tamaño (48 x $37 \mathrm{~cm})$ (fig. 7).

\section{VALORACIÓN Y SÍNTESIS}

Una vez descritas las aplicaciones de material latericio en las termas de Campo Valdés, procederemos a exponer una valoración ordenada de las mo- 


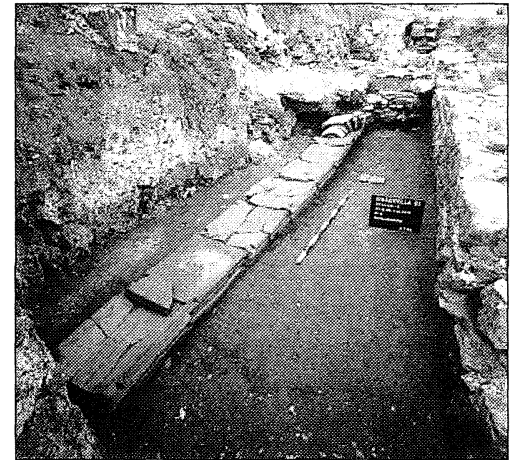

Figura 7.-Conducción de traída de aguas al frigidarium.

dalidades identificadas, sus aplicaciones y sus peculiaridades en relación a los modelos canónicos y a las soluciones adoptadas en otras termas hispanas (Fernández Ochoa, Morillo, Zarzalejos, 1995, e.p.).

- Bessalis: Ladrillo cuadrado de dos tercios de pie (unos 19,7 cm de lado) que Vitrubio (De Arch., $\mathrm{V}, 10$ ) asocia a la realización de las pilae que soportaron la suspensura de los hypocausta. En las termas gijonesas se encuentra relacionado con esta función canónica en los tepidaria (T1 y T2) y en el caldarium (C). Con un uso diverso, se emplean también en la fábrica de muros refractarios en el apodyterium (A) y en el caldarium (C). Cuando las termas dejan de utilizarse como tales, encontramos este tipo de ladrillo reutilizado para enrasar la piscina del frigidarium.

La aplicación canónica de los lateres bessales está constatada en numerosos conjuntos balnearios hispanos, según hemos indicado en otro lugar (Fernández Ochoa, Morillo, Zarzalejos, 1995, e.p.). Este hecho no hace sino confirmar que el uso primordial de estas piezas fue el que se recoge en las indicaciones vitrubianas.

- Pedalis o Tetradoron: Ladrillo de formato cuadrado, de un pie de lado (unos 29,6 cm). Según Vitrubio, este later era adecuado para la realización de basas y capiteles de las pilas de sustentación de la suspensura. Con esta misma finalidad sólo se documenta en Gijón en las pilae de la sudatio circular (S). No obstante, volvemos a encontrarlos formando parte de algunas pilas de los arquillos del hipocausto de la sudatio rectangular (S?), o en los muretes del praefurnium de esta misma estancia.

En el conjunto de las termas hispanas no resulta excesivamente frecuente el empleo de basas y capiteles en las pilae. Esta observación puede estar mediatizada por el estado de conservación de los edificios, aunque existen complejos mejor conservados en los que el latericio usado como capitel o basa de las pilae escapa al canon clásico del later pedalis.

- Sesquipedalis: Ladrillo cuadrado de un pie y medio de lado (unos $44,4 \mathrm{~cm}$ ), cuyo uso recomendado por las normas vitrubianas era servir como pavimento del area del hipocausto. Con este uso canónico no ha sido identificado ningún pavimento de area en las termas de Campo Valdés, como tampoco en el resto de Hispania. Debe concluirse que estos lateres se hallan ausentes de los edificios balnearios hispanos, por razones probablemente de abaratamiento de los costes, según hemos indicado en otro lugar (Fernández Ochoa, Morillo, Zarzalejos, 1995, e.p.).

- Bipedalis: Ladrillo cuadrado de dos pies de lado (unos $59,2 \mathrm{~cm}$ ). Vitrubio propone entre sus usos su integración en el sistema de sustentación de la suspensura. En las termas de Gijón le hallamos desempeñando esta función en el tepidarium (T1) y en la sudatio circular (S). En el primero de los casos, no se ha documentado ningún ejemplar in situ, pero es relativamente fácil adivinar su existencia a partir de cálculos sobre las distancias de las pilae del hipocausto.

Muchos edificios termales hispanos muestran la aplicación canónica de estos grandes lateres, al tiempo que su gran formato favoreció su empleo en otras partes de las construcciones, como arcos de praefurnia, o en la regularización de paramentos.

- Lydion: Ladrillo rectangular de un pie por un pie y medio (unos 29,6 x 44,4 cm). Fue, sin duda alguna, el módulo latericio más versátil y, por tanto, el más empleado. Las termas gijonesas acreditan sus múltiples usos, puesto que se constata su aplicación en los pasos de calor, bases, muros y arcos de praefurnia, umbrales, etc. Además se identifican en los escalones de la piscina del frigidarium, en los accesos escalonados al apodyterium y al frigidarium, en las bases y cubiertas de algunas canalizaciones y, como material reutilizado, en el enrasamiento tardío de la piscina antes citada. Esta multiplicidad de usos encuentra reflejo en buena parte de los establecimientos termales hispanos, donde aparecen indistintamente empleados en cualquiera de las partes estructurales de las habitaciones calientes.

- Cuneati: Ladrillo en forma de cuña, con sección trapezoidal, habitualmente destinado a la realización de arcos y bóvedas. Con esta función se han documentado en los arquillos del hipocausto del apodyterium (A) y en los de la sudatio rectangular (S?). Ha sido posible comprobar su presencia en muros refractarios y muretes de canalización.

- Ladrillos troncopiramidales: Piezas cerámi- 
cas empleadas a modo de plinto en forma de ábaco en pilas de arquillos. Esta modalidad no resulta frecuente, hasta el momento, fuera de la región cantábrica. Además del caso gijonés, donde se han constatado en el hipocausto de la sudatio rectangular (S?), están presentes también en las termas existentes bajo la catedral de Santander (Casado Soto-González Echegaray, 1985).

- Ladrillos circulares: Se utilizan exclusivamente para la realización de las pilae de los hipocaustos. En las termas de Campo Valdés se encuentran formando la mayor parte de las pilas de la sudatio circular (S). Asimismo las pilae del pequeño hipocausto levantado al sur de la citada sudatio, se componen de ladrillos circulares y rectangulares alternativamente.

- Ladrillos rectangulares: No responden a un módulo estándar y sus aplicaciones en Gijón se reducen a la realización de muros refractarios.

- Ladrillos romboidales: Su presencia es muy escasa, dado que tan sólo ha aparecido un ejemplar fuera de contexto.

- Tubuli latericii: Piezas huecas con perforaciones, que se utilizan exclusivamente en la construcción de las concamerationes. En Gijón se han localizado in situ en la sudatio circular (S) y en la rectangular (S?). En ambos casos, se trata de piezas prismáticas huecas, con orificios circulares en los lados estrechos (fig. 8a).

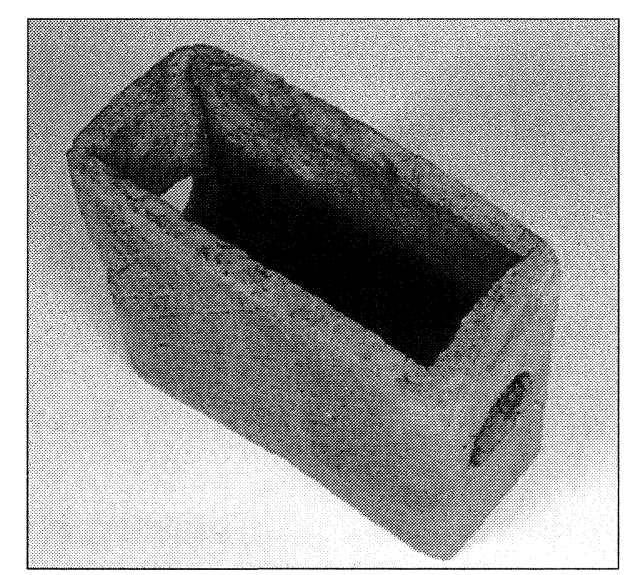

Figura 8a.-Tubulus de la concameratio de la posible sudatio rectangular.

- Tegulae e imbrices: Además de su normal utilización en la cubierta de los edificios, ambos tipos se aplicaron con frecuencia en las estructuras hidráulicas o de saneamiento, dadas las aptitudes formales de tegulae e imbrices para formar la base y la cubierta de los canales. En las termas de Cam- po Valdés se emplearon tegulae en la base de la mayor parte de las canalizaciones y también como cubrición de una de ellas. En otras ocasiones los usos son menos canónicos; así sucede en el hipocausto de la sudatio rectangular ( $\mathrm{S}$ ?) donde una tegula sirve para regular el arranque del arco del praefurnium. Entre las piezas de Gijón se han documentado dos modelos con tamaños diferentes. Uno de ellos presenta grandes dimensiones (46/49 × 37 $\mathrm{cm})$ y se adapta a la tónica constatada en regiones vecinas como Gallaecia, donde la media se sitúa en torno a 49,8 x 37,6 cm (Pérez Losada, 1992, 248). El segundo tipo, de hallazgo menos frecuente, se corresponde con piezas de proporciones inferiores $(46 \times 18 \mathrm{~cm})$. Su relación con la infraestructura hidráulica, apuntada como hipótesis por Alvargonzález (Alvargonzález, 1965, 19), se confirma ahora mediante su localización in situ formando parte de una conducción.

Las imbrices, además de formar parte de la suspensura, se emplearon también como base y cubierta de algunas canalizaciones. En ciertos casos, se utilizaron imbrices de gran tamaño $(51 \times 24 \times 8 \mathrm{~cm})$ como base de la conducción. Otras piezas, de menores dimensiones $(46 \times 17 \mathrm{~cm})$, se hallaron formando parte de la cubierta de otro canal. Asimismo se ha comprobado la existencia de piezas de sección apuntada en la base del canal relacionable con uno de los tepidaria (T1). Estas mismas imbrices se identificaron en otras construcciones romanas gijonesas, como el aliviadero del aljibe de la Plaza de Jovellanos (Fernández Ochoa, 1994, 43, lám. XIII), o un desagüe de la muralla tardía descubierto en la zona inmediata a la iglesia de San Pedro.

- Marcas de officina: Los trabajos de reexcavación de las termas de Campo Valdés no han proporcionado piezas selladas con indicación de origen. Por el contrario, se recuperó un ladrillo rectangular con el numeral XLII (fig. 8b), realizado

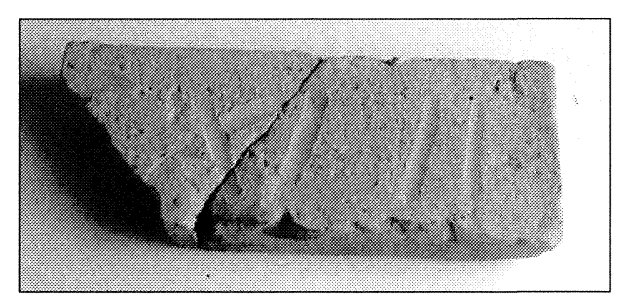

Figura 8b.-Fragmento de ladrillo con inscripción numérica.

con los dedos antes de la cocción. Las inscripciones numerales sobre material latericio se identifican tradicionalmente con marcas de control. Su presen- 

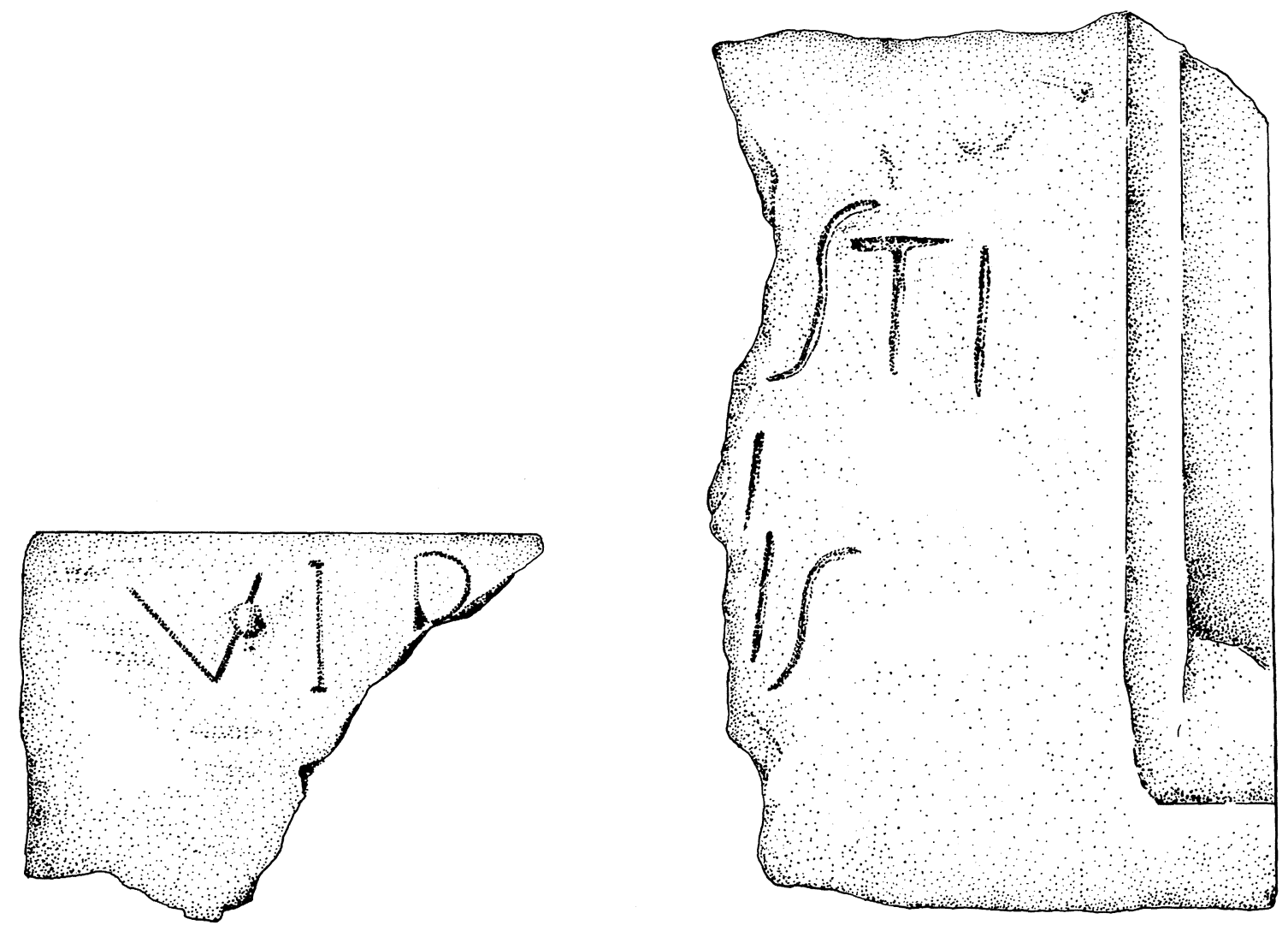

1

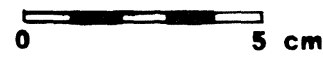

2

Figura 9.-Fragmentos de latericio con inscripción.

cia en Hispania no es muy abundante, si bien en Itálica se han localizado varias piezas (Roldán, 1993, 119 y 255, fig. 55). En un fragmento de otro ladrillo se lee $V I[R][\ldots]$ en inscripción incisa realizada antes de la cocción (fig. 9,1). Un fragmento de $t e$ gula reproduce el extremo derecho de un texto compuesto por al menos tres registros: [...] STI/[...]I/ [...]IS (fig. 9,2). También los trabajos antiguos realizados por Alvargonzález en las termas produjeron ejemplares inscritos; entre ellos debemos destacar un ladrillo cuneatus perteneciente a un arco, hoy desaparecido, situado en el costado norte de la sudatio rectangular (S?), con leyenda griega en cursiva interpretada por Fita como $L e$ (gio) IV M(acedónica) (Alvargonzález, 1965, 17, lám. IX). Hoy día esta lectura se considera muy dudosa, no sólo por razones epigráficas sino de índole histórica (González Echegaray-Solana, 1975, 176-7). En la propia ciudad de Gijón, la factoría de salazones excavada en la Plaza del Marqués proporcionó una mar- ca retrógrada sobre tegula en la que se lee Licini, relacionada con otros sellos hallados en yacimientos asturianos (Fernández Ochoa, 1994, 60, fig.45, 227).

En síntesis, y en función de todas las características anotadas, cabría extraer alguna otra consideración de índole más general. La aplicación de latericio en las termas gijonesas se adapta a los usos habituales en este tipo de edificios romanos. A través de nuestro análisis se demuestra el empleo de módulos canónicos acordes con las prescripciones vitrubianas ( $D e$ Arch., V, 10) y con los modelos en uso dentro y fuera del territorio hispano (Brodribb, 1987, passim.). Aún en aquellos casos cuyas soluciones escapan a la propuesta del tratadista - realización de las areae con opus signinum frente al uso de sesquipedalis-, hemos constatado que el fenómeno se produce en numerosos ejemplos hispanos (Fernández Ochoa, Morillo, Zarzalejos, 1995, e.p.), sin que ello deba considerarse menoscabo de la «ro- 
manidad» canónica de los edificios. Obviamente, las termas de Campo Valdés constituyen un caso modélico de la adopción del concepto de baño romano, gestado en la Campania y transferido después al resto del orbe romano. Esta adopción implica la implantación de una costumbre que se plasma en modelos arquitectónicos concretos y sistemas constructivos netamente romanos en territorios donde la tradición edicilia era pétrea y ajena a la versatilidad del later coctus. Las termas de Gijón reflejan, tanto como otros restos exhumados en el casco viejo de la ciudad, el marcado índice de integración de la sociedad astur en las nuevas fórmulas de vida y costumbres romanas. Este fenómeno no se produce localizadamente en el territorium de la antigua Gijón, sino que se manifiesta a través de diversos testimonios que se incrementan a medida que la investigación arqueológica avanza en todo el noroeste peninsular (Fernández Ochoa, 1993 b; Fernández Ochoa - Morillo, 1994).

En otro orden de cosas, nuestra aportación pretende sumarse al despegue reciente de los estudios sobre edilicia hispanorromana (Bermúdez, 1988; Roldán, 1992 y 1993; Pérez Losada, 1992 a y b). El caudal informativo contenido en descripciones completas de los materiales constructivos empleados en los edificios romanos aporta nuevas posibilidades a la investigación. En este sentido, el estudio del latericio conlleva argumentos para la identificación funcional de los ambientes termales, permite precisar la estratigrafía horizontal del edificio y determinar fases constructivas. Asimismo, el latericio puede ser fuente de conocimiento sobre aspectos concretos de la producción y comercialización de materiales cerámicos de uso constructivo.

Esta vía de análisis, apenas cultivada en la Arqueología Hispanorromana, ofrece un punto de partida para estudios comparativos de los que pueden derivar nuevas interpretaciones de los edificios $y$, en consecuencia, aportaciones renovadas al campo histórico.

\section{BIBLIOGRAFÍA}

AlvargonZÁlez, C., 1965: Las termas romanas de Campo de Valdés (Gijón), Gijón.

Bermúdez, A., 1998: Materiales cerámicos de construcción empleados en la arquitectura romana de Tárraco y su entorno: producción, difusión, consumo y aplicación, Tesis Doctoral, Universidad de Barcelona.
Bonet, J. A., 1970: Biografía de la villa y puerto de Gijón, Gijón.

BrodribB, G., 1987: Roman brick and tile, Gloucester.

Casado Soto, J. M. y González Echegaray, J., 1985: «CRISTO. Excavaciones en la Parroquia del Santísimo. Arqueología», Gran Enciclopedia de Cantabria, III, Santander.

Degbomont, J. M., 1984: Hypocaustes. Le chauffage par hypocauste dans l'habitat privé, Lieja.

Fernández OCHOA, C., 1992: «La muralla romana de Gijón», Los orígenes de Gijón, Gijón.

FERNÁNDEZ OCHOA, C., 1993: «Arqueología romana de Gijón. Balance de una década de excavaciones», Trabalhos de Antropologia y Etnologia, 34 (1-3), 365-380.

FERNÁNDEZ OCHOA, C., 1993 b: «La ciudad en los territorios septentrionales de la Península ibérica», La ciudad hispanorromana, Tarragona.

Fernández OChOA, C., 1994: Una industria de salazones romana en la plaza del Marqués (Gijón, Asturias), Gijón.

Fernández OchoA, C. y Morillo, A., 1994: De Brigantium a Oiasso. Aproximación a los enclaves marítimos cantábricos en época romana, Madrid.

Fernández OchoA, C. (Dir.), 1995: Las termas romanas de Campo Valdés, en Astures (Guía de la Exposición), Gijón.

Fernández Ochoa, C.; Morillo, A.; Zarzalejos, M., 1995: «Material latericio en las termas romanas de Hispania», Actas de la Table Ronde La Brique et ses dérivés à l'époque romaine. Production, utilisation et diffusion dans les provinces Occidentales (Hispanie, Gaule et Italie), Madrid.

GonZález Echegaray, J. y Solana, J. M., 1975: «La Legio IV Macedónica en España», Hispania Antiqua, V, 151-203.

Nielsen, I., 1990: Thermae et balnea, Aarhus.

PÉrez LosADA, F., 1992: «Contribución ó estudio da cerámica de construcción na Galicia Romana (I)», Galicia: da romanidade á xermanización. Problemas históricos e culturais, 241-261.

PÉrez LosAdA, F., 1992 b: «Hipocaustos na Galaecia Romana», Gallaecia, 13, 129-176.

RoldÁN, L., 1992: Técnicas constructivas romanas en Carteia, Monografías de Arquitectura Romana, 1, Madrid.

RoldÁn, L., 1993: Técnicas constructivas romanas en Itálica (Santiponce, Sevilla), Monografías de Arquitectura Romana, 2, Madrid.

SomozA, J., 1908: Gijón en la Historia general de Asturias, vol. I, Gijón (Ed. 1971). 